PROCEEDINGS OF THE

AMERICAN MATHEMATICAL SOCIETY

Volume 139, Number 10, October 2011, Pages 3393-3403

S 0002-9939(2011)10774-3

Article electronically published on May 12, 2011

\title{
BOUNDEDNESS FOR SURFACES IN WEIGHTED PROJECTIVE 4-SPACES
}

\author{
L. V. RAMMEA AND G. K. SANKARAN \\ (Communicated by Ted Chinburg)
}

\begin{abstract}
Ellingsrud and Peskine in 1989 proved that there exists a bound on the degree of smooth nongeneral type surfaces in $\mathbb{P}^{4}$. The latest proven bound is 52 by Decker and Schreyer in 2000 .

In this paper we consider bounds on the degree of a quasismooth nongeneral type surface in weighted projective 4 -space. We show that such a bound in terms of the weights exists and compute an explicit bound in simple cases.
\end{abstract}

\section{INTRODUCTION}

Ellingsrud and Peskine EP proved that there exists an integer $d_{0}$ such that all smooth nongeneral type surfaces in $\mathbb{P}^{4}$ have degree less than or equal to $d_{0}$. This motivated a search for such surfaces, partly by computational methods, and also an effort to find an effective bound on $d_{0}$, begun by Braun and Fløystad in [BF]. As far as we know the smallest proven bound is 52 by Decker and Schreyer [DS].

Some of the methods used to find such surfaces are also applicable to surfaces in weighted projective spaces $\mathbb{P}^{4}(w)$ (some first steps in this direction are taken in [Ra]). It is therefore natural to ask whether a similar bound can be found for the degree of quasismooth nongeneral type surfaces in a weighted projective space with given weights. In this paper we show that such a readily computable bound (of course depending on the weights) does exist, and we compute it in some cases.

To show that a bound exists, all we need is a fairly simple adaptation of the way in which the results of $[\mathrm{EP}$ ( or $[\mathrm{BF}$ ) are applied. For a computable bound we use the results of $[\mathrm{BF}]$ together with some information about the contribution from the singularities of the surface in $\mathbb{P}^{4}(w)$.

Our procedure is to exploit the representation of $\mathbb{P}^{4}(w)$ as a quotient of $\mathbb{P}^{4}$ by a finite group action. Starting with a quasismooth nongeneral type surface $X$ in weighted projective 4 -space $\mathbb{P}^{4}(w)$, we take its cover in $\mathbb{P}^{4}$. This will (usually) be of general type, but it will have invariants bounded in terms of those of $X$, and the results of $[\mathrm{BF}]$ still apply in this situation.

Received by the editors November 7, 2009.

2010 Mathematics Subject Classification. Primary 14M07; Secondary 14J25.

This work forms part of the Bath Ph.D. thesis of the first author, supported by a Commonwealth Scholarship of the Association of Commonwealth Universities.

(C)2011 American Mathematical Society Reverts to public domain 28 years from publication 


\section{Bounding the DEGREes}

We fix weights $w=\left(w_{0}, w_{1}, w_{2}, w_{3}, w_{4}\right)$ with $w_{i} \in \mathbb{N}$; unless otherwise stated, $i$ and $j$ always denote indices in the range $0 \leq i, j \leq 4$. We may assume that any four of the $w_{i}$ are coprime; such weights are called well-formed (see [Do, 1.3.1] and $[\mathrm{I}-\mathrm{F}, 5.9 \& 5.11])$. We also order the weights so that $w_{i} \leq w_{i+1}$; in particular, the largest weight is $w_{4}$. We write $|w|$ for the sum of the weights, and $m$ for their product. The weighted projective space $\mathbb{P}^{4}(w)$ of dimension 4 is defined to be the quotient $\left(\mathbb{C}^{5} \backslash\{0\}\right) / \mathbb{C}^{*}$, where $\mathbb{C}^{*}$ acts by

$$
t:\left(x_{0}, \ldots, x_{4}\right) \rightarrow\left(t^{w_{0}} x_{0}, \ldots, t^{w_{4}} x_{4}\right)
$$

A surface $X \subset \mathbb{P}^{4}(w)$ is said to be quasismooth if its punctured affine cone $X^{*}$ is smooth, that is, if $X^{*}=q^{-1}(X)$ is smooth, where $q:\left(\mathbb{C}^{5} \backslash\{0\}\right) / \mathbb{C}^{*} \rightarrow \mathbb{P}^{4}(w)$ is the quotient map; see [Do, 3.1.5] or [I-F, 6.3].

Alternatively $([\overline{\mathrm{Do}}, 1.2 .2])$ we may regard $\mathbb{P}^{4}(w)$ as a quotient of $\mathbb{P}^{4}$ under an action of the group $G_{w}=\prod_{i} \mathbb{Z} / w_{i} \mathbb{Z}$ of order $m$. A generator $g_{i}$ of the $i$ th factor acts by $x_{i} \mapsto x_{i}^{w_{i}}$. We denote the quotient map $\mathbb{P}^{4} \rightarrow \mathbb{P}^{4}(w)$ by $\phi_{w}$.

Suppose that $X$ is a quasismooth surface, not of general type, in $\mathbb{P}^{4}(w)$. Denote by $\hat{X}$ the cover of $X$ in $\mathbb{P}^{4}$ under the $m$-to- 1 map $\phi_{w}$; then $\hat{X}$ is smooth. We always assume that $X$ and $\hat{X}$ are nondegenerate; that is, $\hat{X}$ is not contained in any hyperplane in $\mathbb{P}^{4}$.

Let $f: \tilde{X} \rightarrow X$ be the minimal resolution of $X$ (note that $\tilde{X}$ need not be a minimal surface):

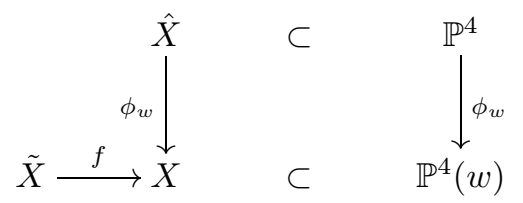

Further let $d$ be the degree of $X \subset \mathbb{P}^{4}(w)$ and $\pi$ the sectional genus of $X$. These are defined as follows: $\mathbb{P}^{4}(w)$ and $X$ are $\mathbb{Q}$-factorial varieties and there are $\mathbb{Q}$-line bundles $\mathcal{O}_{\mathbb{P}^{4}(w)}(1), \mathcal{O}_{X}(1)$ and $K_{X}$. Writing $H$ for the class of $\mathcal{O}_{X}(1)$ in Pic $X \otimes \mathbb{Q}$ and using the intersection form on Pic $X$ we have $d=H^{2}$ and $2 \pi-2=H \cdot\left(H+K_{X}\right)$, so $d, \pi \in \mathbb{Q}$.

We let $\hat{d}$ be the degree of $\hat{X}$ and $\hat{\pi}$ the sectional genus of $\hat{X}$. We put

$$
\hat{s}=\min \left\{k \mid h^{0} \mathcal{I}_{\hat{X}}(k) \neq 0\right\}
$$

and denote by $\sigma_{f}$ the number of irreducible exceptional curves of $f$.

We first collect the facts about these invariants of the smooth surface $\hat{X} \subset \mathbb{P}^{4}$.

Proposition 1.1. If $\hat{X} \subset \mathbb{P}^{4}$ is a smooth surface (possibly of general type), and $r \leq \hat{s}$ and $r^{2}<\hat{d}$, then

$$
2 \hat{\pi} \leq \frac{\hat{d}^{2}}{r}+(r-4) \hat{d}+1
$$

Moreover

$$
\hat{d}^{2}-5 \hat{d}-10(\hat{\pi}-1)+12 \chi\left(\mathcal{O}_{\hat{X}}\right)-2 K_{\hat{X}}^{2}=0
$$


Finally, if $\hat{d}>\hat{s}(\hat{s}-1)$, we have the lower bound for $\chi\left(\mathcal{O}_{\hat{X}}\right)$ :

$$
\begin{aligned}
\chi\left(\mathcal{O}_{\hat{X}}\right) \geq & \frac{\hat{d}^{3}}{6 \hat{s}}+\hat{d}^{2} \frac{\hat{s}-5}{4 \hat{s}}+\hat{d} \frac{3 \hat{s}^{2}-30 \hat{s}+71}{24} \\
& -\frac{\hat{s}^{4}-5 \hat{s}^{3}-\hat{s}^{2}+5 \hat{s}}{24}-\frac{\gamma^{2}}{2}-\gamma\left(\frac{\hat{d}}{\hat{s}}+s-\frac{5}{2}\right),
\end{aligned}
$$

where $0 \leq \gamma \leq \hat{d}(\hat{s}-1)^{2} /(2 \hat{s})$.

Proof. The inequality (1.1) is a consequence of EP, (B), (C), page 2]. Let $\hat{H}$ denote a general hyperplane section of $\hat{X}$, so that $\hat{\pi}=g(\hat{H})$. According to Ro] (as quoted in EP, (C), page 2]), if $\hat{s}>r$ and $\hat{d}>r^{2}$, then $\hat{H} \subset \mathbb{P}^{3}$ does not lie on any surface of degree $<r$. Therefore, according to [EP, (B), page 2], we have $r(2 \hat{\pi}-2) \leq \hat{d}^{2}+r(r-4) \hat{d}$. If $\hat{s}=r$, then (again by [EP, (B), page 2$]$ ) we have the same inequality because then $\hat{H}$ does lie on a surface of degree $r$.

Equation (1.2) is the double point formula as stated in $[\mathrm{EP}$ and $\mathrm{BF}$. The estimate (1.3) is $[\mathrm{BF},(1.1)(\mathrm{e})]$.

A more precise version of (1.1), valid under certain conditions, is given in $\mathrm{BF}$, (1.1)]. In order to bound the degree of smooth surfaces in $\mathbb{P}^{4}$, what is needed is not the precise form of (1.3) but an estimate of the form $\chi\left(\mathcal{O}_{\hat{X}}\right) \geq a(\hat{s}) \hat{d}^{3}+o\left(\hat{d}^{3}\right)$, where $a(\hat{s})$ is some positive constant depending on $\hat{s}$ only. Ellingsrud and Peskine proved the existence of such a bound in $[\mathrm{EP}$ but did not give an explicit one.

It will be convenient to work with the invariants $c_{1}^{2}(S)=K_{S}^{2}$ and $c_{2}(S)$ (which is the topological Euler number $e(S)$ ) of a smooth projective surface $S$ : these are connected by Noether's formula

$$
12 \chi\left(\mathcal{O}_{S}\right)=c_{1}^{2}(S)+c_{2}(S) .
$$

Since we are assuming that $\tilde{X}$ is not of general type we have (as in $[\mathrm{EP}]$ and $[\mathrm{BF}]$ ) that $K_{\tilde{X}}^{2} \leq 9$. Moreover, unless $\tilde{X}$ is a rational surface with $K_{\tilde{X}}^{2} \geq 6$ we also have $6 \chi\left(\mathcal{O}_{\tilde{X}}\right) \geq K_{\tilde{X}}^{2}$ (i.e. $\left.c_{2}(\tilde{X})-c_{1}^{2}(\tilde{X}) \geq 0\right)$. If $\tilde{X}$ is a rational surface, then $\chi\left(\mathcal{O}_{\tilde{X}}\right)=1$, so $c_{2}(\tilde{X})-c_{1}^{2}(\tilde{X})=12 \chi\left(\mathcal{O}_{\tilde{X}}\right)-2 K_{\tilde{X}}^{2}=12-2 K_{\tilde{X}}^{2} \geq-6$. So in any case if $X$ is not of general type we have

$$
c_{1}^{2}(\tilde{X})-c_{2}(\tilde{X}) \leq 6 .
$$

So we need to estimate $\hat{d}$ and $\hat{\pi}$ in terms of $d$ and $\pi$, and $K_{\hat{X}}^{2}$ and $\chi\left(\mathcal{O}_{\hat{X}}\right)$ in terms of $K_{\tilde{X}}^{2}$ and $\chi\left(\mathcal{O}_{\tilde{X}}\right)$. We shall show the two propositions below.

Proposition 1.2. Suppose $X$ is a quasismooth normal surface in $\mathbb{P}^{4}(w)$. Then

$$
c_{1}^{2}(\hat{X}) \leq m c_{1}^{2}(\tilde{X})+\theta_{1},
$$

where, writing $\hat{\delta}=2 \hat{\pi}-2-\hat{d}$, we have

$$
\theta_{1}=k_{0}+k_{1} \hat{d}+k_{2} \hat{\delta}
$$

for suitable $k_{0}, k_{1}, k_{2}$ depending only on the weights $w_{i}$. Moreover

$$
c_{2}(\hat{X}) \geq m c_{2}(\tilde{X})-\theta_{2},
$$

and

$$
\theta_{1}+\theta_{2}=k_{0}^{\prime}+k_{1}^{\prime} \hat{d}+k_{2}^{\prime} \hat{\delta}
$$

for suitable $k_{0}^{\prime}, k_{1}^{\prime}, k_{2}^{\prime}$ depending only on the weights, and $k_{2}^{\prime}>-5$. 
This proposition will be proved in Sections 3 and 4 , below.

Our main qualitative result is then the following.

Theorem 1.3. There exists $d_{w} \in \mathbb{N}$ depending only on the weights $w_{i}$ such that any quasismooth normal surface $X \subset \mathbb{P}^{4}(w)$ of degree $d>d_{w}$ is of general type.

Proof. We have seen that $\hat{X} \rightarrow X$ is $m$-to- 1 , so

$$
\hat{d}=m d,
$$

so it is sufficient to show that if $X$ is not of general type, then $\hat{d}$ is bounded by a function of the weights.

Suppose then that $X$ is not of general type. We have, by adjunction, $2 \hat{\pi}-2=$ $\hat{H} \cdot\left(\hat{H}+K_{\hat{X}}\right)=\hat{d}+\hat{\delta}$, where $\hat{H}$ is a hyperplane section of $\hat{X}$. Therefore by the estimate (1.1) we obtain

$$
\hat{\delta} \leq \frac{1}{r} \hat{d}^{2}+(r-5) \hat{d}
$$

as long as $r \leq \hat{s}$ and $r^{2}<\hat{d}$. We may also write the double point formula as

$$
\hat{d}^{2}-10 \hat{d}-5 \hat{\delta}+c_{2}(\hat{X})-c_{1}^{2}(\hat{X})=0 .
$$

By Proposition 1.2 and the inequality (1.5) we have

$$
c_{2}(\hat{X})-c_{1}^{2}(\hat{X}) \geq-6 m-\left(\theta_{1}+\theta_{2}\right),
$$

so

$$
\begin{aligned}
0 & \geq \hat{d}^{2}-10 \hat{d}-5 \hat{\delta}-6 m-\left(\theta_{1}+\theta_{2}\right) \\
& =\hat{d}^{2}-\left(10+k_{1}^{\prime}\right) \hat{d}-\left(6 m+k_{0}^{\prime}\right)-\left(5+k_{2}^{\prime}\right) \hat{\delta} .
\end{aligned}
$$

Combining this with (1.11) gives (since $5+k_{2}^{\prime}>0$ )

$$
\begin{aligned}
0 & \geq \hat{d}^{2}-\left(10+k_{1}^{\prime}\right) \hat{d}-\left(6 m+k_{0}^{\prime}\right)-\left(5+k_{2}^{\prime}\right)\left(\frac{1}{r} \hat{d}^{2}+(r-5) \hat{d}\right) \\
& =\left(1-\frac{5+k_{2}^{\prime}}{r}\right) \hat{d}^{2}-\left(\left(10+k_{1}^{\prime}+\left(5+k_{2}^{\prime}\right)(r-5)\right) \hat{d}-\left(6 m+k_{0}^{\prime}\right) .\right.
\end{aligned}
$$

So if $\hat{s}>k_{2}^{\prime}+5$ we may take $r=k_{2}^{\prime}+6$ and this bounds $\hat{d}$ in that case.

On the other hand, suppose that $X$ is not of general type and $\hat{s} \leq k_{2}^{\prime}+5$. Then using Noether's formula, the double point formula (1.12), and (1.3) we have

$$
\begin{aligned}
0 & =\hat{d}^{2}-10 \hat{d}-5 \hat{\delta}+12 \chi\left(\mathcal{O}_{\hat{X}}\right)-2 c_{1}^{2}(\hat{X}) \\
& \geq-2 c_{1}^{2}(\hat{X})+\frac{2}{\hat{s}} \hat{d}^{3}+O\left(\hat{d}^{2}\right) \\
& \geq-2 m c_{1}^{2}(\tilde{X})-\theta_{1}+\frac{2}{\hat{s}} \hat{d}^{3}+O\left(\hat{d}^{2}\right) \\
& \geq \frac{2}{\hat{s}} \hat{d}^{3}+O\left(\hat{d}^{2}\right)-18 m-k_{0}-k_{1} \hat{d}-k_{2} \hat{\delta} \\
& =\frac{2}{\hat{s}} \hat{d}^{3}+O\left(\hat{d}^{2}\right)
\end{aligned}
$$

by (1.7) and (1.11); the constants depend on $\hat{s}$, but this is now bounded in terms of the weights. So again we obtain a bound for $\hat{d}$ in terms of the $w_{i}$. 


\section{Singularities of $\mathbb{P}^{4}(w)$ And of $X$}

In this section we collect some preliminary information about the action of $G_{w}$ on $\mathbb{P}^{4}$ and on $\hat{X}$. We choose an isomorphism $G_{w} \rightarrow \prod \mathbb{Z} / w_{i} \mathbb{Z}$ by choosing generators $g_{i} \in G_{w}$ of order $w_{i}$. The singularities arise at fixed points of the $G_{w}$-action, so let us consider those.

Suppose that $x=\left(x_{0}: \ldots: x_{4}\right) \in \mathbb{P}^{4}$ is fixed by $g=g_{0}^{a_{0}} \ldots g_{4}^{a_{4}}$. Without loss of generality we take $x_{0}=1$; then for $j \neq 0$ we have $\zeta_{0}^{-a_{0}} \zeta_{j}^{a_{j}}=1$, where $\zeta_{j}=e^{2 \pi i / w_{j}}$.

Lemma 2.1. If $x \in \mathbb{P}^{4}$ is fixed by a nontrivial element of $G_{w}$, then $x$ lies in a coordinate linear subspace $\mathbb{P}_{J}$ given by $\mathbb{P}_{J}=\left\{x_{j}=0 \mid j \in J \subset\{0, \ldots, 4\}\right\}$. The stabiliser of a general point of $\mathbb{P}_{J}$ is the group $\Gamma_{J}$ generated by the $g_{j}$ for $j \in J$ and the element $g_{J}=\prod_{i \notin J} g_{i}^{w_{i} / r_{J}}$, where $r_{J}=\operatorname{hcf}\left(a_{i} \mid i \notin J\right)$.

This is immediate from the description of the action above. By a general point in $\mathbb{P}_{J}$ is meant, in this case, a point that is not in $\mathbb{P}_{J^{\prime}}$ for any $J^{\prime} \supset J$.

Lemma 2.2. The singularities of $X$ are cyclic quotient singularities whose order divides one of the weights.

Proof. At a fixed point $x \in \mathbb{P}^{4}$, the elements $g_{j} \in \Gamma_{J}$ act on the tangent space by quasi-reflections: the $j$ th eigenvalue is $\zeta_{j}^{a_{j}}$ and the others are 1 . So the quotient by the subgroup $\Gamma_{J}^{\prime}$ generated by those elements is smooth, and the singularity of $\mathbb{P}_{w}$ or of $X$ at $z=\phi_{w}(x)$ is a quotient by the action of the cyclic group generated by $g_{J}$. The order of this element, or of its image in $\Gamma_{J} / \Gamma_{J}^{\prime}$, is $r_{J}$, which divides $w_{i}$ for $i \notin J$.

Remark 2.3. If $\# J=1$, then $r_{J}=1$ since the weights are well-formed, so the general point of a coordinate hyperplane in $\mathbb{P}^{4}(w)$ is smooth. For each $i$, the number of singular points of $X$ with $z_{i}=0$ is at most $\hat{d}$.

Remark 2.4. If the weights are pairwise coprime, then the singularities occur at the points $P_{0}=(1: 0: \ldots: 0), \ldots, P_{4}=(0: \ldots: 0: 1) \in \mathbb{P}^{4}(w)$, and the singularity of $\mathbb{P}_{w}$ at $P_{i}$ has order exactly $w_{i}$. If $X \ni P_{i}$, then $X$ also has a cyclic quotient singularity of order $w_{i}$ at $P_{i}$.

Lemma 2.5. Suppose that $(Y, 0)$ is a nondegenerate smooth surface germ in $\left(\mathbb{A}^{4}, 0\right)$ with coordinates $t_{1}, \ldots, t_{4}$ at $0 \in \mathbb{A}^{4}$. Let $\gamma$ be the quasi-reflection $\gamma\left(t_{1}\right)=\xi t_{1}$, where $\xi$ is a primitive nth root of unity, and let $Y$ be $\gamma$-invariant. Then $Y$ meets $A=\left(t_{1}=0\right)$ tranversely.

Proof. Suppose not; then $T_{Y, 0} \subset A$. Therefore the ideal $\mathcal{I}_{Y, 0} \subset \mathcal{O}_{\mathbb{A}^{4}, 0}$ contains an element $f$ of the form $f=t_{1}+h$ with $h \in \mathfrak{m}^{2} \subset \mathcal{O}_{\mathbb{A}^{4}, 0}$, where $\mathfrak{m}$ is the maximal ideal of $\mathcal{O}_{\mathbb{A}^{4}, 0}$.

We write $h=\sum_{\nu=0}^{n-1} h_{\nu}$, where $\gamma\left(h_{\nu}\right)=\xi^{\nu}\left(h_{\nu}\right)$. If we write $h$ as a polynomial in $t_{1}$ so $h=\sum_{r} a_{r}\left(t_{2}, t_{3}, t_{4}\right) t_{1}^{r}$, we have $h_{\nu}=\sum_{r \equiv \nu} a_{\bmod n} a_{r}\left(t_{2}, t_{3}, t_{4}\right) t_{1}^{r}$. Then

$$
\mathcal{I}_{Y, 0} \ni\left(1+\gamma+\gamma^{2}+\cdots+\gamma^{n-1}\right)(f)=n h_{0},
$$

so $\mathcal{I}_{Y, 0} \ni f-h_{0}=t_{1}+\sum_{\nu \neq 0} h_{\nu}$. But $t_{1}$ divides the right-hand side, so since $h \in \mathfrak{m}^{2}$ we have $f-h_{0}=t_{1}(1+b)$, where $b \in \mathfrak{m}$. Since $\mathcal{I}_{Y, 0}$ is a prime ideal contained in $\mathfrak{m}$, this implies $t_{1} \in \mathcal{I}_{Y, 0}$, contradicting the nondegeneracy. 
Corollary 2.6. If $w_{i} \neq 1$, then $\hat{X}$ meets the ramification divisor $\mathbb{P}_{\{i\}}$ transversely and the curve $\hat{C}_{i}=\hat{X} \cap \mathbb{P}_{\{i\}}$ is a smooth curve of genus $\hat{\pi}$.

Proof. The second part follows immediately from the first, which is immediate from Lemma 2.5

\section{COMPARING $c_{1}^{2}$}

In this section we prove (1.6) and (1.7) from Proposition 1.2 and give values for the constants $k_{0}, k_{1}$ and $k_{2}$.

Let $\Delta=\sum_{1 \leq \nu \leq \sigma_{f}} a_{\nu} E_{\nu}$ be the discrepancy of $f$, so that $a_{\nu} \in \mathbb{Q}$ and $K_{\tilde{X}}=$ $f^{*} K_{X}+\Delta$. Then $f^{*} K_{X} \cdot \Delta$ vanishes and $\left(f^{*} K_{X}\right)^{2}=K_{X}^{2}$, so $K_{\tilde{X}}^{2}=K_{X}^{2}+\Delta^{2}$.

Lemma 3.1. If $f_{0}: \tilde{Y} \rightarrow Y$ is the minimal resolution of an isolated cyclic quotient $(Y, 0)$ of order $n$ and the discrepancy of $f_{0}$ is $\Delta_{0}$, then $0>\Delta_{0}^{2} \geq-n$.

Proof. This (which is not a sharp bound) is most easily seen by toric methods. If the singularity is $\frac{1}{n}(1, a)$ with $(n, a)=1$, then the minimal resolution is described by taking the decomposition given by the convex hull of $\mathbb{Z}^{2}+\frac{1}{n}(1, a) \mathbb{Z}$ in the first quadrant of $\mathbb{R}^{2}$. The exceptional curves $E_{\nu}, 0<\nu<k$, correspond to primitive vectors $P_{\nu}=\left(x_{\nu}, y_{\nu}\right)$ of this lattice: put $\ell_{\nu}=x_{\nu}+y_{\nu}$, and write $E_{0}$ and $E_{k}$ for the toric curves corresponding to the rays spanned by $(1,0)$ and $(0,1)$. Then we have $E_{\nu} E_{\nu \pm 1}=1$ and $E_{\mu} E_{\nu}=0$ if $\mu \neq \nu, \nu \pm 1$. Moreover, on $\tilde{Y}$ we have $\sum_{0 \leq \nu \leq k} \ell_{\nu} E_{\nu} \equiv 0$ (linear equivalence) and $\Delta=-\sum_{0<\nu<k} E_{\nu}$. Therefore

$$
\begin{aligned}
\Delta_{0}^{2} & =\sum_{0<\nu<k} E_{\nu}\left(\sum_{0<\mu<k} E_{\mu}\right) \\
& =\sum_{0<\nu<k} E_{\nu}\left(\left(\sum_{\mu \neq 0, \nu, k} E_{\mu}\right)+E_{\nu}\right) \\
& =\sum_{0<\nu<k} E_{\nu}\left(-E_{0}-E_{k}+\sum_{\mu \neq \nu}\left(1-\frac{\ell_{\mu}}{\ell_{\nu}}\right) E_{\mu}\right) \\
& =-2-\sum_{0<\nu<k}\left(\left(\frac{\ell_{\nu-1}}{\ell_{\nu}}-1\right)+\left(\frac{\ell_{\nu+1}}{\ell_{\nu}}-1\right)\right) .
\end{aligned}
$$

Suppose for definiteness that $\ell_{n u+1}>\ell_{\nu}$. Then $\frac{\ell_{\nu+1}}{\ell_{\nu}}-1$ is twice the area (relative to the lattice $\Lambda=\mathbb{Z}^{2}+\frac{1}{n}(1, a) \mathbb{Z}$ ) of the triangle $T_{\nu}^{+}=P_{\nu} Q_{\nu} P_{\nu+1}$, where $Q_{\nu}=\frac{\ell_{\nu+1}}{\ell_{\nu}} P_{\nu}$, since Area $\left(O P_{\nu} P_{\nu+1}\right)=\frac{1}{2}$ relative to $\Lambda$. So

$$
\begin{aligned}
-\frac{1}{2} \Delta_{0}^{2} & \leq-1-\sum_{0<\nu<k} \operatorname{Area}\left(T_{\nu}^{+}\right) \\
& =-\operatorname{Area}\left(O P_{0} P_{1}\right)-\operatorname{Area}\left(O P_{k-1} P_{k}\right)-\sum_{0<\nu<k} \operatorname{Area}\left(T_{\nu}^{+}\right) .
\end{aligned}
$$

But these triangles do not overlap and they are contained in the unit triangle $O P_{0} P_{k}$, which has area $\frac{n}{2}$ relative to $\Lambda$. 
Now we compute $K_{\hat{X}}^{2}$ from $K_{\hat{X}}=\phi_{w}^{*}\left(K_{X}\right)+\sum\left(w_{i}-1\right) \hat{H}_{i}$, where $\hat{H}_{i}=\mathbb{P}_{\{i\}} \cap \hat{X}=$ $\left(x_{i}=0\right)$, and so we get

$$
K_{\hat{X}}^{2}=m K_{X}^{2}+2 \sum\left(w_{i}-1\right) \hat{\delta}-\sum\left(w_{i}-1\right)\left(w_{j}-1\right) \hat{d}
$$

since $\phi_{w}^{*}\left(K_{X}\right)^{2}=m K_{X}^{2}$.

Proposition 3.2. We have $c_{1}^{2}(\hat{X}) \leq m c_{1}^{2}(\tilde{X})+\theta_{1}$, where (recall that $w_{4}$ is the largest weight)

$$
\theta_{1}=\left(10 m w_{4}-\sum_{0 \leq i, j \leq 4}\left(w_{i}-1\right)\left(w_{j}-1\right)\right) \hat{d}+2(|w|-5) \hat{\delta} .
$$

Proof. For a singular point $z \in \operatorname{Sing}(X)$ we denote the discrepancy at $z$ by $\Delta_{z}$. If $z \in H_{J}=\phi_{w}\left(\mathbb{P}_{J}\right) \cap X$, then the order of the singularity is $r_{J}=\operatorname{hcf}\left(w_{i} \mid i \notin J\right)$. There are at most $\left(\begin{array}{l}5 \\ 2\end{array}\right) \hat{d}$ distinct points on the $\hat{H}_{\{i j\}}$ altogether, so the total number of singular points is at most $10 \hat{d}$.

Each singular point has order $r_{J}$ dividing some of the $w_{i}$, so $\Delta_{z}^{2} \geq-r_{J} \geq-w_{4}$. Then

$$
\begin{aligned}
c_{1}^{2}(\tilde{X})=K_{\tilde{X}}^{2} & =K_{X}^{2}+\Delta^{2} \\
& =K_{X}^{2}+\sum_{z \in \operatorname{Sing}(X)} \Delta_{x}^{2} \\
& \geq K_{X}^{2}-10 w_{4} \hat{d} .
\end{aligned}
$$

Now, using (3.1), we get

$$
\begin{aligned}
c_{1}^{2}(\hat{X}) & =m K_{X}^{2}+2 \hat{\delta}(|w|-5)-\hat{d} \sum_{0 \leq i, j \leq 4}\left(w_{i}-1\right)\left(w_{j}-1\right) \\
& \leq m c_{1}^{2}(\tilde{X})+2 \hat{\delta}(|w|-5)+\hat{d}\left(10 m w_{4}-\sum_{0 \leq i, j \leq 4}\left(w_{i}-1\right)\left(w_{j}-1\right)\right),
\end{aligned}
$$

as required.

If the $w_{i}$ are pairwise coprime we can do slightly better. In that case the only singularities are at the points $P_{i}$ if they are in $X$. Therefore we have

$$
c_{1}^{2}(\tilde{X})=K_{X}^{2}+\sum_{P_{i} \in X} \Delta_{i}^{2} \geq K_{X}^{2}-\sum_{i} q_{i} w_{i}
$$

where $\Delta_{i}$ is the discrepancy at $P_{i}$ and $q_{i}=1$ if $P_{i} \in X, q_{i}=0$ if $P_{i} \notin X$. This gives

$$
c_{1}^{2}(\hat{X}) \leq m c_{1}^{2}(\tilde{X})+m \sum q_{i} w_{i}+2(|w|-5) \hat{\delta}-\hat{d} \sum_{0 \leq i, j \leq 4}\left(w_{i}-1\right)\left(w_{j}-1\right) .
$$

\section{COMPARING $c_{2}$}

Recall that if $x \in \hat{X} \cap \mathbb{P}_{J}$, then $\Gamma_{J}$ stabilises $x$. We put $\hat{X}_{J}=\hat{X} \cap\left(\mathbb{P}_{J} \backslash \bigcup_{J^{\prime} \supset J} \mathbb{P}_{J^{\prime}}\right)$. On $\hat{X}_{J}$ the stabiliser is precisely $\Gamma_{J}$. The order of $\Gamma_{J}$ is $h_{J}=r_{J} \prod_{j \in J} w_{j}$; in particular, $h_{\emptyset}=1$ and $h_{\{i\}}=w_{i}$.

$\hat{X}_{\{i\}}$ is the complement of up to $4 \hat{d}$ points on a smooth curve of genus $\hat{\pi}$, by Corollary 2.6. Those points lie in some $\hat{X}_{J}$ with $\# J \geq 2$ : in particular they all lie 
on $\hat{H}_{j}$ for some $j \neq i$, and there are $\hat{d}$ such points for each such $j$. They may not all be distinct, however. Therefore

$$
2-2 \hat{\pi}>e\left(\hat{X}_{\{i\}}\right) \geq 2-2 \hat{\pi}-4 \hat{d} .
$$

Denote by $\mathcal{Q}$ the set of points of $\hat{X}$ lying in at least two coordinate hyperplanes of $\mathbb{P}^{4}$ : thus $\mathcal{Q}=\hat{X} \cap \bigcup_{\# J \geq 2} \mathbb{P}_{J}$ as a set. The set $\mathcal{Q}$ is finite, of cardinality $q \leq 10 \hat{d}$, and $\hat{X}=\hat{X}_{\emptyset} \sqcup \bigcup_{i} \hat{X}_{\{i\}} \sqcup \mathcal{Q}$.

We put $X_{J}=\phi_{w}\left(\hat{X}_{J}\right)$, for $J \subset\{0, \ldots, 4\}$, so that

$$
\left.\phi_{w}\right|_{\hat{X}_{J}}: \hat{X}_{J} \longrightarrow X_{J}
$$

is unramified and its degree is $\left|G_{w}: \Gamma_{J}\right|=m / r_{J}$.

Lemma 4.1. For each $x \in \mathcal{Q}$, let $r_{x}$ be the order of the singularity of $z=\phi_{w}(x) \in$ $X$, so $r_{x}=r_{J}$ if $x \in \hat{X}_{J}$. Then

$$
c_{2}(\tilde{X}) \leq e(X)+\sum_{x \in \mathcal{Q}}\left(r_{x}-1\right) .
$$

Proof. The resolution $f: \tilde{X} \rightarrow X$, in a neighbourhood of $z$, consists of a sequence of at most $r_{x}-1$ blowups, needed to resolve the quotient singularity of order $r_{x}$ at $z \in X$. Therefore $\sigma_{f} \leq \sum_{x \in \mathcal{Q}}\left(r_{x}-1\right)$. Each blowup contracts a smooth rational curve: topologically, therefore, $f$ contracts $\sigma_{f} 2$-spheres to points, and each of these contractions reduces the Euler characteristic by 1 , so $e(\tilde{X})=e(X)+\sigma_{f} \leq$ $e(X)+\sum_{x \in \mathcal{Q}}\left(r_{x}-1\right)$.

Proposition 4.2. We have $c_{2}(\hat{X}) \geq m c_{2}(\tilde{X})-\theta_{2}$, where (recall that $w_{4}$ is the largest weight)

$$
\theta_{2}=\left(10 m w_{4}-(|w|-5)\right) \hat{d}-(|w|-5) \hat{\delta}
$$

Proof. By the additivity of the Euler characteristic we have

$$
\begin{aligned}
c_{2}(\hat{X}) & =\sum_{J} e\left(\hat{X}_{J}\right) \\
& =\sum_{J}\left|G_{w}: \Gamma_{J}\right| e\left(X_{J}\right) \\
& =m e\left(X_{\emptyset}\right)+\sum_{J \neq \emptyset}\left|G_{w}: \Gamma_{J}\right| e\left(X_{J}\right) \\
& =m\left(e(X)-\sum_{J \neq \emptyset} e\left(X_{J}\right)\right)+\sum_{J \neq \emptyset}\left|G_{w}: \Gamma_{J}\right| e\left(X_{J}\right) \\
& =m e(X)+\sum_{J \neq \emptyset}\left(1-h_{J}\right)\left|G_{w}: \Gamma_{J}\right| e\left(X_{J}\right) \\
& =m e(X)+\sum_{J \neq \emptyset}\left(1-h_{J}\right) e\left(\hat{X}_{J}\right) .
\end{aligned}
$$


Write $h_{x}=h_{J}$ if $x \in \hat{X}_{J}$. Using $\hat{X}=\hat{X}_{\emptyset} \sqcup \bigcup_{i} \hat{X}_{\{i\}} \sqcup \mathcal{Q}$ and Lemma 4.1, this gives

$$
\begin{aligned}
c_{2}(\hat{X}) & =m e(X)-\sum_{i}\left(w_{i}-1\right) e\left(\hat{X}_{\{i\}}\right)-\sum_{x \in \mathcal{Q}}\left(h_{x}-1\right) \\
& \geq m c_{2}(\tilde{X})-\sum_{i}\left(w_{i}-1\right) e\left(\hat{X}_{\{i\}}\right)-m \sum_{x \in \mathcal{Q}}\left(r_{x}-1\right)-\sum_{x \in \mathcal{Q}}\left(h_{x}-1\right) \\
& \geq m c_{2}(\tilde{X})-(|w|-5)(2-2 \hat{\pi})-m \sum_{x \in \mathcal{Q}}\left(r_{x}-1\right)-\sum_{x \in \mathcal{Q}}\left(h_{x}-1\right) \\
& =m c_{2}(\tilde{X})+(|w|-5)(\hat{d}+\hat{\delta})-(m+1) \sum_{x \in \mathcal{Q}}\left(r_{x}-1\right) \\
& \geq m c_{2}(\tilde{X})+(|w|-5)(\hat{d}+\hat{\delta})-10 m w_{4} \hat{d},
\end{aligned}
$$

as claimed, since $q \leq 10 \hat{d}, r_{x} \leq w_{4}$ and $h_{x} \leq m$.

We can now complete the proof of Proposition 1.2, and hence of Theorem 1.3, by remarking that from Propositions 3.2 and 4.2 we get

$$
\theta_{1}+\theta_{2}=\left(20 m w_{4}-(|w|-5)-\sum\left(w_{i}-1\right)\left(w_{j}-1\right)\right) \hat{d}+(|w|-5) \hat{\delta},
$$

so $k_{2}^{\prime}=|w|-5>-5$.

\section{EXAmples}

It would of course be possible to obtain an explicit bound as in Theorem 1.3 from the argument above. However, such a bound would be likely to be rather poor. In specific cases it is possible to obtain a bound better than the general one implied above. Although we still do not expect such a bound to be good, in the sense that we expect that in fact all nongeneral type surfaces will be of much lower degree, in some cases it is not absurdly big.

Example 5.1 (weights $(1,1,1,1,2)$ ). We calculate a bound for the case of weights $(1,1,1,1,2)$. In this case there is at most one singular point of $X$, and if there is a singular point it is an ordinary double point. We let $q$ be the number of singularities of $X$, so $q=0$ or $q=1$.

In this case the singularity, if any, is canonical, and blowing up once gives a crepant resolution, so $\Delta^{2}=0$ and $c_{1}^{2}(\tilde{X})=K_{X}^{2}$. Moreover $K_{\hat{X}}=\phi^{*} K_{X}+\hat{H}$, so

$$
\begin{aligned}
c_{1}^{2}(\hat{X}) & =\left(\phi^{*} K_{X}+\hat{H}\right)^{2} \\
& =2 K_{X}^{2}+2 \phi^{*} K_{X} \hat{H}+\hat{H}^{2} \\
& =2 c_{1}^{2}(\tilde{X})+2\left(K_{\hat{X}}-\hat{H}\right) \hat{H}+\hat{H}^{2} \\
& =2 c_{1}^{2}(\tilde{X})-\hat{d}+2 \hat{\delta} .
\end{aligned}
$$

We also have $c_{2}(\tilde{X})=e(X)+q$ and

$$
\begin{aligned}
c_{2}(\hat{X}) & =2 e(X)-\sum_{i}\left(w_{i}-1\right) e\left(\hat{X}_{\{i\}}\right)-\sum_{x \in \mathcal{Q}}\left(h_{x}-1\right) \\
& =2 e(X)-(2-2 \hat{\pi})-q \\
& =2 c_{2}(\tilde{X})+\hat{d}+\hat{\delta}-3 q .
\end{aligned}
$$


Thus $\theta_{1}=-\hat{d}+2 \hat{\delta}$ and $\theta_{2}=3 q-\hat{d}-\hat{\delta}$. Therefore $k_{0}^{\prime}=3 q, k_{1}^{\prime}=-2$ and $k_{2}^{\prime}=1$, and (1.14) and the formula below it give

$$
0 \geq\left(1-\frac{6}{r}\right) \hat{d}^{2}-(6 r-22) \hat{d}-(12+3 q)
$$

as long as $r \geq \hat{s} \geq 7$ and $r^{2}<\hat{d}$. Taking $r=7$, we see that $\hat{d} \leq 140$ in this case. (By taking $r=9$ we can obtain $\hat{d} \leq 96$, but as we shall see that will not yield a better bound in the end. Clearly, taking $r \geq 10$ we cannot do better than $\hat{d} \leq 100$ because for this case we need $r^{2}<\hat{d}$.)

We must also deal with the cases $\hat{s}<7$ : if we use $r=9$ we must also handle $\hat{s}=7$ and $\hat{s}=8$ separately. But now we have, using $c_{1}^{2}(\tilde{X}) \leq 9$, the estimate (1.1) for $\hat{\delta}$ with $r=\hat{s}$, the bounds on $\chi\left(\mathcal{O}_{\hat{X}}\right)$ and $\gamma$ from Proposition 1.1, and the double point formula

$$
\begin{aligned}
0= & \hat{d}^{2}-10 \hat{d}+12 \chi\left(\mathcal{O}_{\hat{X}}\right)-2 c_{1}^{2}(\hat{X}) \\
= & \hat{d}^{2}-10 \hat{d}+12 \chi\left(\mathcal{O}_{\hat{X}}\right)-4 c_{1}^{2}(\tilde{X})+2 \hat{d}=4 \hat{\delta} \\
\geq & \hat{d}^{2}-8 \hat{d}+12 \chi\left(\mathcal{O}_{\hat{X}}\right)-36-\frac{4}{\hat{s}} \hat{d}^{2}-4(\hat{s}-5) \hat{d}+4 \\
\geq & 12\left[\frac{\hat{d}^{3}}{6 \hat{s}}+\hat{d}^{2}\left(\frac{\hat{s}-5}{4 \hat{s}}\right)+\hat{d}\left(\frac{3 \hat{s}^{2}-30 \hat{s}+71}{24}\right)-\frac{\hat{s}^{4}-5 \hat{s}^{3}-\hat{s}^{2}+5 \hat{s}}{24}\right. \\
& \left.-\frac{1}{2}\left(\frac{(\hat{s}-1)^{4}}{4 \hat{s}^{2}}\right) \hat{d}^{2}-\left(\frac{(\hat{s}-1)^{2}}{2 \hat{s}^{2}}\right) \hat{d}^{2}-\left(\frac{(\hat{s}-5 / 2)(\hat{s}-1)^{2}}{2 \hat{s}}\right) \hat{d}\right] \\
& +\hat{d}^{2}\left(1-\frac{4}{\hat{s}}\right)+\hat{d}(-8-4(\hat{s}-5))-32 \\
= & \frac{2}{\hat{s}} \hat{d}^{3}-\frac{3 \hat{s}^{4}-12 \hat{s}^{3}+22 \hat{s}^{2}+2 \hat{s}+15}{2 \hat{s}^{2}} \hat{d}^{2} \\
& -\frac{9 \hat{s}^{3}-16 \hat{s}^{2}-23 \hat{s}-30}{2 \hat{s}} \hat{d}-\frac{\hat{s}^{4}-5 \hat{s}^{3}-\hat{s}^{2}+5 \hat{s}+64}{2}
\end{aligned}
$$

(for $\hat{s}=2$, the $-\left(\frac{(\hat{s}-5 / 2)(\hat{s}-1)^{2}}{2 \hat{s}}\right) \hat{d}$ term should be omitted). It is easy to compute that this implies $\hat{d} \leq 91$ for $\hat{s} \leq 6$, but for $\hat{s}=7$ we obtain only $\hat{d} \leq 153$, so taking $r=9$ does not improve the overall bound. Taking $r=7$, we find the overall bound $\hat{d} \leq 140$.

Generally we see from (1.3) that for large weights, and hence large $\hat{s}$, the two biggest terms in absolute value in the cubic will be the $\hat{d}^{3}$ term and a term $-\frac{\hat{s}^{2}}{4} \hat{d}^{2}$. Therefore the bound on $\hat{d}$ will be around $|w|^{3} / 8$.

Example 5.2 (Weights $(1,1,1,2,6)$ ). As a further example, we calculate a bound for weights $(1,1,1,2,6)$. In this case the possible singularities are: up to $\hat{d}$ order 2 singularities (ordinary nodes) along $x_{0}=x_{1}=x_{2}=0$, with $r_{x}=h_{x}=2$, and one singularity of order 6 at $(0: 0: 0: 0: 1)$, with $r_{x}=6, h_{x}=12$. At the double points, $\Delta_{x}^{2}=0$, and at the 6 -fold point one has in fact $\Delta_{x}^{2} \geq-\frac{8}{3}$. 
In this case we have $K_{\hat{X}}^{2}=12 K_{x}^{2}-36 \hat{d}+12 \hat{\delta}$ and $c_{1}^{2}(\tilde{X})=K_{X}^{2}-\sum_{x} \Delta_{x}^{2} \geq K_{x}^{2}-\frac{8}{3}$, so $\theta_{1}=32-36 \hat{d}+12 \hat{\delta}$. We also have

$$
\begin{aligned}
c_{2}(\hat{X}) & =12 c_{2}(\tilde{X})+6(\hat{d}+\hat{\delta})-12 \sum_{x \in \mathcal{Q}}\left(r_{x}-1\right)-\sum_{x \in \mathcal{Q}}\left(h_{x}-1\right) \\
& \geq 12 c_{2}(\tilde{X})+6(\hat{d}+\hat{\delta})-12 \hat{d}-60-\hat{d}-11 \\
& =2 c_{2}(\tilde{X})-71-7 \hat{d}+6 \hat{\delta} .
\end{aligned}
$$

Thus $\theta_{2}=71+7 \hat{d}-6 \hat{\delta}$. Therefore $k_{0}^{\prime}=103, k_{1}^{\prime}=-29$ and $k_{2}^{\prime}=6$, and the quadratic is

$$
0 \geq\left(1-\frac{11}{r}\right) \hat{d}^{2}-(11 r-274) \hat{d}-175
$$

as long as $r \geq \hat{s} \geq 12$ and $r^{2}<\hat{d}$. Taking $r=12$, we see that $\hat{d} \leq 699$ in this case.

We must also deal with the cases $\hat{s}<12$ by using the cubic. For $\hat{s}=11$ we obtain $\hat{d} \leq 710$; as this is already bigger than 699 it is no use looking at other choices for $r$. Smaller values of $\hat{s}$ give smaller bounds, so the overall bound remains $\hat{d} \leq 710$.

\section{REFERENCES}

BF. R. Braun, G. Fløystad, A bound for the degree of smooth surfaces in $\mathbb{P}^{4}$ not of general type. Compos. Math. 93 (1994), 211-229. MR1287697 (95g:14037)

DS. W. Decker, F.-O. Schreyer, Non-general type surfaces in $\mathbb{P}^{4}$ : some remarks on bounds and constructions. J. Symbolic Comput. 29 (2000), 545-582. MR1769655 (2002a:14064)

Do. I. Dolgachev, Weighted projective varieties. In: Group actions and vector fields (Vancouver, B.C., 1981), 34-71, Lecture Notes in Math. 956, Springer, Berlin, 1982. MR704986 (85g:14060)

EP. G. Ellingsrud, C. Peskine, Sur les surfaces lisses de $\mathbb{P}_{4}$. Invent. Math. 95 (1989), 1-11. MR969410 (89j:14023)

I-F. A. Iano-Fletcher, Working with weighted complete intersections. In: Explicit birational geometry of 3-folds, 101-173. London Math. Soc. Lecture Notes 281, Cambridge University Press, Cambridge 2000. MR.1798982 (2001k:14089)

Ra. L.V. Rammea, Computations and bounds for surfaces in weighted projective four-spaces. Ph.D. thesis, Bath, 2009.

Ro. L. Roth, On the projective classification of surfaces. Proc. London Math. Soc. (2) 42, 142-170 (1936).

Department of Mathematics and Computer Science, The National University of Lesotho, P.O. Roma 180, Lesotho

E-mail address: lv.rammea@nul.1s

Department of Mathematical Sciences, University of Bath, Bath BA2 7Ay, United KINGDOM

E-mail address: G.K.Sankaran@bath.ac.uk 